International Journal of Algebra, Vol. 1, 2007, no. 3, 145 - 149

\title{
On the Set of Gaps in Numerical Semigroups
}

\author{
Sedat ILHAN \\ Department of Mathematics, Faculty of Science and Art \\ Dicle University, Diyarbakır 21280, Turkey \\ sedati@dicle.edu.tr
}

\begin{abstract}
In this paper, we give some results on the set of gaps in numerical semigroups. Also, we determine $S$ by the set of gaps $H(S)$.
\end{abstract}

Mathematics Subject Classification: 20M14, 20F50

Keywords: Numerical semigroups, gaps, Apéry set

\section{Introduction}

Let $\mathbb{N}=\{0,1,2, \cdots n, \cdots\}$ and $S \subseteq \mathbb{N}$. $S$ is called a numericalsemigroup if $S$ is sub-semigroup of $(\mathbb{N},+)$ with $0 \in S$.

It is known that every numerical semigroup is finitely generated, i.e. there exist elements of $S$, say $n_{0}, n_{1}, \cdots, n_{p}$ such that $n_{0}<n_{1}<\cdots<n_{p}$ and

$$
S=\left\langle n_{0}, n_{1}, \cdots, n_{p}\right\rangle=\left\{\sum_{i=0}^{p} k_{i} n_{i} \quad: k_{i} \in \mathbb{N}\right\}
$$

and

$$
\text { G.C.D. }\left(n_{0}, n_{1}, \cdots, n_{p}\right)=1 \Leftrightarrow \operatorname{Card}(\mathbb{N} \backslash S)<\infty
$$

by $[1]$.

Let us give the following definitions known for numerical semigroup $S$.

$g(S)=\max \{x \in \mathbb{Z}: x \notin S\}$ is called the Frobenius number of $S$, where $\mathbb{Z}$ is the integer set. Thus, $S$ numerical semigroup is $S=\left\{0, n_{0}, n_{1}, \cdots, g(S)+\right.$ $1 \rightarrow \cdots\}$ ( The arrow " $\rightarrow$ " means that every integer which is greater then $g(S)+1$ belongs to $S)$.

We say that a numerical semigroup is symmetric if for every $x \in \mathbb{Z} \backslash S$, we have $g(S)-x \in S$ by [2].

For $n \in S \backslash\{0\}$, we define the Apéry set of the element $n$ as the set 


$$
A p(S, n)=\{s \in S: s-n \notin S\} .
$$

It can easily be proved that $A p(S, n)$ is formed by the smallest elements of $S$ belonging to the different congruence classes modn. Thus, $\sharp(A p(S, n))=n$ and $g(S)=\max (A p(S, n))-n$, where $\sharp(A)$ stands for cardinality $(A)$ by [3]. The elements of $\mathbb{N} \backslash S$, denote by $H(S)$, are called gaps of $S$. A gap $x$ of a numerical semigroup $S$ is fundamental if $\{2 x, 3 x\} \subset S$. We denote by $F H(S)$ the set of fundamental gaps of $S$.

Let $D\left(x_{i}\right)=\left\{x \in \mathbb{N}: x \mid x_{i}, \exists i \in\{1,2, \ldots, r\}\right\}$. Since $S$ is a numerical semigroup and, $i \in\{1,2, \ldots, r\}$, for $x_{i} \in F H(S)$ we write $S=\mathbb{N} \backslash D\left(x_{i}\right)$ by [4]. If $S=\left\langle s_{1}, s_{2}\right\rangle$, then

$$
A p\left(S, s_{1}\right)=\left\{\left(s_{1}-r\right) s_{2}: r=1,2, \cdots, s_{1}\right\}
$$

and

$$
A p\left(S, s_{2}\right)=\left\{\left(s_{2}-r\right) s_{1}: r=1,2, \cdots, s_{2}\right\}
$$

the set of gaps of $S$ is

$$
H(S)=\{0,1,2, \ldots, g(S)\} \backslash\left(A p\left(S, s_{1}\right) \bigcup A p\left(S, s_{2}\right) \bigcup\left\{s_{1}+s_{2}\right\}\right)
$$

by $[5]$.

\section{Main Results}

In this section, we give some results for the set of gaps of $S$.

Theorem 1. If $S_{1}$ and $S_{2}$ are two numerical semigroups, then we write

$$
H\left(S_{1} \cap S_{2}\right)=H\left(S_{1}\right) \bigcup H\left(S_{2}\right) .
$$

Proof. We have $a \in H\left(S_{1} \bigcap S_{2}\right)$.It follows easily that $a \in H\left(S_{1}\right) \cup H\left(S_{2}\right)$. Since

$$
a \in H\left(S_{1} \bigcup S_{2}\right) \Longrightarrow a \notin\left(S_{1} \bigcap S_{2}\right) \Longrightarrow\left\{\begin{array}{cc}
(i) & a \in S_{1}, a \notin S_{2} \\
(i i) & a \notin S_{1}, a \in S_{2} \\
(\text { iii) } & a \notin S_{1}, a \notin S_{2}
\end{array}\right\} .
$$

Conversely,let us assume that $b \in H\left(S_{1}\right) \cup H\left(S_{2}\right)$. Since $b \in H\left(S_{1}\right) \cup H\left(S_{2}\right) \Longrightarrow$ $b \in H\left(S_{1}\right) \vee H\left(S_{2}\right)$, we obtain the required result by definition of gaps.

Corollary. For $1 \leq i \leq n, S_{i}$ are numerical semigroups, then we can write

$$
H\left(\bigcap_{i=1}^{n} S_{i}\right)=\bigcup_{i=1}^{n} H\left(S_{i}\right) .
$$

Theorem 2. If $S_{1}$ and $S_{2}$ are two numerical semigroups such that $S_{1} \nsubseteq S_{2}$ or $S_{2} \nsubseteq S_{1}$, then we have

$$
F H\left(S_{1} \bigcap S_{2}\right)=F H\left(S_{1}\right) \cup F H\left(S_{2}\right) .
$$


Proof. If $x \in F H\left(S_{1} \cap S_{2}\right) \Longleftrightarrow x \in H\left(S_{1} \cap S_{2}\right) \wedge\{2 x, 3 x\} \subset\left(S_{1} \cap S_{2}\right)$, then $x \in F H\left(S_{1}\right) \bigcup F H\left(S_{2}\right)$ by definition of fundamental gaps.

Corollary. If $S_{i}$ are numerical semigroups such that $S_{i} \nsubseteq S_{j}$ and $S_{j} \nsubseteq S_{i}$, where $1 \leq i, j \leq n$, then we can write

$$
F H\left(\bigcap_{i=1}^{n} S_{i}\right)=\bigcup_{i=1}^{n} F H\left(S_{i}\right) .
$$

Theorem 3. Let $S_{1}$ and $S_{2}$ are two numerical semigroups. $K=S_{1} \bigcap S_{2}$ numerical semigroup is determined as follows

$$
K=S_{1} \cap S_{2}=\mathbb{N} \backslash D\left(x_{j}\right)=\mathbb{N} \backslash\left(H\left(S_{1}\right) \bigcup H\left(S_{2}\right)\right),
$$

where $x_{j} \in F H\left(S_{1}\right) \bigcap F H\left(S_{2}\right), j \in\{1,2, \ldots, r\}$.

Proof.We find the required result by Theorem 1 and Theorem 2 .

Example 1. Let

$$
S_{1}=\langle 3,5,7\rangle=\{0,3,5,6,7,8, \rightarrow, \cdots\}
$$

and

$$
S_{2}=\langle 4,5,11\rangle=\{0,4,5,8,9,10,11,12, \rightarrow, \cdots\} .
$$

be two numerical semigroups. Thus,we find that

$$
\begin{gathered}
S_{1} \cap S_{2}=\{0,5,8,9,10, \rightarrow, \cdots\}, \\
H\left(S_{1}\right)=\{1,2,4\}, H\left(S_{2}\right)=\{1,2,3,6,7\}, F H\left(S_{1}\right)=\{4\} \text { and } F H\left(S_{2}\right)=
\end{gathered}
$$
$\{6,7\}$. Hence, we obtain that

$$
H\left(S_{1} \bigcap S_{2}\right)=H\left(S_{1}\right) \cup H\left(S_{2}\right)=\{1,2,3,4,6,7\}
$$

and

$$
F H\left(S_{1} \cap S_{2}\right)=F H\left(S_{1}\right) \bigcup F H\left(S_{2}\right)=\{4,6,7\} .
$$

Moreover, we write $D(4,6,7)=\{1,2,3,4,6,7\}$. Finally, we determine

$$
K=S_{1} \cap S_{2}=\{0,5,8,9,10, \rightarrow, \cdots\}
$$

numerical semigroup as follows

$$
K=S_{1} \bigcap S_{2}=\{0,5,8,9,10, \rightarrow, \cdots\}=\mathbb{N} \backslash D(4,6,7)=\mathbb{N} \backslash\left(H\left(S_{1}\right) \bigcup H\left(S_{2}\right)\right) .
$$

\section{Example 2.Let}

$$
S_{1}=\langle 4,5\rangle=\{0,4,5,8,9,10,12,13, \rightarrow, \cdots\}
$$

and

$$
S_{2}=\langle 3,5\rangle=\{0,3,5,6,8,9, \rightarrow, \cdots\} .
$$


be two numerical semigroups.In this case,

$$
\begin{gathered}
A p\left(S_{1}, 4\right)=\{5(4-r): r=1,2,3,4\}=\{0,5,10,15\}, \\
A p\left(S_{1}, 5\right)=\{4(5-r): r=1,2,3,4,5\}=\{0,4,8,12,16\}
\end{gathered}
$$

and $g\left(S_{1}\right)=11$. Hence, we write

$$
\left.H\left(S_{1}\right)=\{0,1,2, \ldots, 11\} \backslash A p\left(S_{1}, 4\right) \bigcup A p\left(S_{1}, 5\right) \bigcup\{9\}\right)=\{1,2,3,6,7,11\}
$$

and $F H\left(S_{1}\right)=\{6,7,11\}$. If we apply the same operations for $S_{2}$ above, then we find that

$$
A p\left(S_{2}, 3\right)=\{5(3-r): r=1,2,3\}=\{0,5,10\}
$$

and

$$
A p\left(S_{2}, 5\right)=\{3(5-r): r=1,2,3,4,5\}=\{0,3,6,9,12\}
$$

and $g\left(S_{2}\right)=7$.

Thus, we write

$$
\left.H\left(S_{2}\right)=\{0,1,2, \ldots, 7\} \backslash A p\left(S_{2}, 3\right) \bigcup A p\left(S_{2}, 5\right) \bigcup\{8\}\right)=\{1,2,4,7\}
$$

and $F H\left(S_{2}\right)=\{4,7\}$.In this case,for $K=S_{1} \bigcap S_{2}=\{0,5,8,9,10,12,13, \rightarrow$ $, \cdots\}$, we obtain

$$
H\left(S_{1} \bigcap S_{2}\right)=H\left(S_{1}\right) \cup H\left(S_{2}\right)=\{1,2,3,4,6,7,11\} .
$$

and

$$
F H\left(S_{1} \cap S_{2}\right)=F H\left(S_{1}\right) \bigcup F H\left(S_{2}\right)=\{4,6,7,11\} .
$$

Moreover, we write $D(4,6,7,11)=\{1,2,3,4,6,7,11\}$ for $4,6,7,11 \in F H\left(S_{1} \bigcap S_{2}\right)$. Thus, we determine numerical semigroup $K=S_{1} \bigcap S_{2}=\{0,5,8,9,10,12,13, \rightarrow$ $, \cdots\}$ as follows:

$$
\begin{gathered}
K=S_{1} \bigcap S_{2}=\mathbb{N} \backslash D(4,6,7,11)=\mathbb{N} \backslash\{1,2,3,4,6,7,11\}= \\
\mathbb{N} \backslash\left(H\left(S_{1}\right) \bigcup H\left(S_{2}\right)\right) .
\end{gathered}
$$

If we apply the same operations for numerical semigroups

$$
S_{1}=\langle 4,5\rangle=\{0,4,5,8,9,10,12,13, \rightarrow, \cdots\}
$$

and

$$
S_{3}=\langle 2,5\rangle=\{0,2,4,5,6,7,8,9, \rightarrow, \cdots\}
$$

Then, we find 


$$
\begin{gathered}
A p\left(S_{3}, 2\right)=\{5(2-r): r=1,2\}=\{0,5\} \\
A p\left(S_{3}, 5\right)=\{2(5-r): r=1,2,3,4,5\}=\{0,2,4,6,8\}
\end{gathered}
$$

and $g\left(S_{3}\right)=3$. Thus, we write

$$
\left.H\left(S_{3}\right)=\{0,1,2,3\} \backslash A p\left(S_{3}, 2\right) \bigcup A p\left(S_{3}, 5\right) \bigcup\{7\}\right)=\{1,3\}
$$

and $F H\left(S_{3}\right)=\{3\}$. In this case, we find that

$$
H\left(S_{1} \cap S_{3}\right)=H\left(S_{1}\right) \bigcup H\left(S_{3}\right)=\{1,2,3,6,7,11\},
$$

but $F H\left(S_{1} \bigcap S_{3}\right)=\{6,7,11\} \neq F H\left(S_{1}\right) \bigcup F H\left(S_{3}\right)$, for numerical semigroup

$$
K=S_{1} \bigcap S_{3}=\{0,4,5,8,9,10,12,13, \rightarrow, \cdots\}=S_{1} .
$$

\section{References}

[1] R. Froberg, C. Gottlieb and R. Haggkvist, On numerical semigroups, Semigroup Forum, 35 (1987), 63-83.

[2] J.C.Rosales and J.I.Garcia- Garcia, Hereditarily Finitely Generated Commutative Monoids , Journal of Algebra, 221 (1999), 723-732.

[3] J.C. Rosales, Numerical Semigroups with Apéry sets of Unique Expression, Journal of Algebra 226 (2000), 479-487 .

[4] J.C. Rosales, P.A. Garcia-Sanchez, J.I.Garcia- Garcia, J.A. Jimenez Madrid,Fundamental gaps in numerical semigroups, Journal of pure and applied algebra, 189 (2004), 301-313.

[5] S. Ilhan, On Apéry sets of Symmetric Numerical Semigroups, International Mathematical Forum, 1, no.10, (2006), 481-484.

\section{Received: July 12, 2006}

